\title{
Revista Colombiana de

\section{Relación entre los atributos del sueño y el cambio en la presión arterial. Análisis de la cohorte CHICAMOCHA}

\author{
Jhon Edgar Vargas ${ }^{a}$, Skarlet Marcell Vásquez ${ }^{a}$, Juan Carlos Villar ${ }^{a, b}$ \\ y Víctor Mauricio Herrera ${ }^{a, *}$
}

\author{
a Grupo de Cardiología Preventiva, Facultad de Ciencias de la Salud, Universidad Autónoma de Bucaramanga, Bucaramanga, \\ Colombia \\ b Departamento de Investigaciones, Fundación Cardioinfantil - Instituto de Cardiología, Bogotá, Colombia
}

Recibido el 26 de diciembre de 2017; aceptado el 1 de septiembre de 2018

Disponible en Internet el 11 de marzo de 2019

\section{PALABRAS CLAVE \\ Sueño; \\ Presión arterial; \\ Hipertensión arterial; \\ Estudios de cohorte; \\ Colombia}

\begin{abstract}
Resumen
Introducción: la hipertensión arterial es responsable de gran parte de la carga de enfermedad cardiovascular; sin embargo, el papel del sueño en su etiología aun es controversial.

Objetivo: determinar la asociación entre atributos del sueño y cambio de la presión arterial. Métodos: estudio de cohorte prospectiva, de base comunitaria, en adultos jóvenes asintomáticos para enfermedad cardiovascular, seguidos por 12,4 años. En la línea de base se determinaron factores sociodemográficos y del comportamiento, y se caracterizó el sueño nocturno usual en términos de calidad, duración y presencia de ronquido. Se realizó medición estandarizada de índices antropométricos y presión arterial. La hipertensión arterial se definió como reporte de tratamiento antihipertensivo o cifras de presión arterial $\geq 140 / 90 \mathrm{~mm} \mathrm{Hg}$.

Resultados: se evaluaron de manera prospectiva 1.032 participantes (media de edad: 36,8 años, $64 \%$ hombres; en la línea de base). La incidencia de hipertensión arterial fue de 15,1\% (IC95\%: $12,8-17,4)$, con evidencia de mayor riesgo en los roncadores, atenuado posteriormente al ajuste por adiposidad $(p>0,05)$. Se observó un gradiente entre la duración del sueño y el cambio en la presión arterial sistólica/diastólica (2,2/1,3 mm Hg en quienes reportaron 6-8 horas/día y 2,7/1,6 $\mathrm{mm} \mathrm{Hg}$ en quienes reportaron $>8$ horas/día, respecto a $<6$ horas/día). Ni la calidad subjetiva del sueño ni el reporte de siesta se asociaron con la presión arterial.

Conclusión: los resultados sugieren que la duración prolongada del sueño podría incrementar la presión arterial en la población.

(C) 2018 Sociedad Colombiana de Cardiología y Cirugía Cardiovascular. Publicado por Elsevier España, S.L.U. Este es un artículo Open Access bajo la licencia CC BY-NC-ND (http:// creativecommons.org/licenses/by-nc-nd/4.0/).
\end{abstract}

\footnotetext{
* Autor para correspondencia.

Correo electrónico: vherrera@unab.edu.co (V.M. Herrera).
} 


\section{KEY WORDS}

Sleep;

Blood pressure;

Arterial hypertension; Cohort studies;

Colombia

\section{Relationship between the attributes of sleep and the change in blood pressure: Analysis of the CHICAMOCHA cohort}

\begin{abstract}
Introduction: Arterial hypertension is responsible for large part of the cardiovascular disease load. However, the role of sleep in its aetiology remains controversial.

Objective: To determine the relationship between sleep attributes and the change in blood pressure.

Methods: A prospective cohort study was carried out on a community basis in young adults asymptomatic for cardiovascular disease, followed up for 12.4 years. At baseline, sociodemographic and behavioural factors were determined. At the same, time nocturnal sleep was characterised in terms of quality, duration and presence of snoring. Standardised anthropometric indices and blood pressure were measured. Arterial hypertension was defined as a report of anti-hypertension treatment or blood pressure figures $\geq 140 / 90 \mathrm{~mm} \mathrm{Hg}$.

Results: A total of 1,032 participants were prospectively evaluated (mean age 36.8 years, $64 \%$ males; at baseline). The incidence of arterial hypertension was $15.1 \%$ (95\% cl: $12.8-17.4$ ), with evidence of a higher risk in snorers, with a subsequent reduction on adjusting for adiposity $(P>.05)$. A gradient was observed between the duration of sleep and the change in systolic/diastolic arterial pressure $(2.2 / 1.3 \mathrm{mmHg}$ in those who reported $6-8$ hours/day, and $2.7 / 1.6 \mathrm{mmHg}$ in those who reported $>8$ hours/day, compared to $<6$ horas/day). Neither the subjective quality of the sleep nor the report of a siesta was associated with the blood pressure. Conclusion: The results suggest that the prolonged duration of sleep could increase blood pressure in the population.

(c) 2018 Sociedad Colombiana de Cardiología y Cirugía Cardiovascular. Published by Elsevier España, S.L.U. This is an open access article under the CC BY-NC-ND license (http:// creativecommons.org/licenses/by-nc-nd/4.0/).
\end{abstract}

\section{Introducción}

La hipertensión arterial es el factor de riesgo que de manera individual explica la mayor carga de mortalidad por enfermedad cardiovascular ${ }^{1}$. En Colombia se estimó en la más reciente Encuesta Nacional de Salud (2007) una prevalencia de hipertensión arterial igual al $23 \%$ en adultos ${ }^{2}$. Esta cifra aproximadamente duplica la prevalencia reportada $(12,3 \%)$ por la segunda Encuesta Nacional de Factores de Riesgo para Enfermedades Crónicas (ENFREC) en 1999³ . Adicionalmente, de acuerdo con el Observatorio Nacional de Salud Pública de Colombia, la hipertensión arterial es la segunda causa de consulta en el país y se confirma que su prevalencia continúa en aumento ${ }^{4}$.

Existe evidencia que relaciona a diferentes alteraciones del sueño, particularmente, la reducción progresiva de su duración durante las últimas décadas, con la incidencia de hipertensión arterial ${ }^{5}$. Estudios de restricción aguda del sueño han identificado el incremento en la actividad del sistema nervioso simpático como uno de los mecanismos que sustenta dicha asociación ${ }^{6,7}$. Lo anterior ha sido confirmado en estudios epidemiológicos, que también sugieren un papel potencial de la duración prolongada del sueño en la hipertensión arterial ${ }^{8}$. Por otra parte, se ha descrito que el ronquido puede aumentar el riesgo de hipertensión arterial debido a la inducción de apnea durante el sueño ${ }^{9}$; sin embargo, la inconsistencia en el hallazgo de apnea en personas que roncan sugiere un efecto independiente del ronquido sobre la presión arterial ${ }^{10}$.
El objetivo de este estudio fue determinar la asociación entre diferentes atributos del sueño y los cambios de presión arterial en adultos jóvenes participantes en la cohorte CHICAMOCHA (Cardiovascular Health Investigation and Collaboration to Assess the Markers and Outcomes of Chagas disease).

\section{Material y métodos}

El estudio CHICAMOCHA es una cohorte de base comunitaria, ensamblada a partir de donantes de sangre representativos de los bancos de sangre de Bucaramanga entre 1997 y 2001. Aunque CHICAMOCHA incluyó, en razón a su objetivo, donantes con pruebas de tamización serológica positivas o negativas para Trypanosoma cruzi (y negativas para el resto de agentes tamizados en bancos de sangre, en relación 1:4), este análisis se circunscribe a los participantes seronegativos de la cohorte. Los datos incluidos aquí se refieren a la visita de línea de base y al seguimiento a este grupo luego de una mediana de 12,4 años.

Los detalles acerca de la metodología de muestreo, selección, contacto y evaluación de los participantes han sido descritos previamente ${ }^{11}$. En breve, todos los donantes de sangre consecutivos, ocasionales y altruistas, con pruebas serológicas negativas de tamización transfusional fueron invitados a participar durante la ventana de tiempo de interés. Las personas entre 18 y 50 años de edad que dieron su consentimiento por escrito y se encontraban aparentemente sanas, fueron entrevistadas por personal del estudio que 
recolectó información sociodemográfica (incluyendo estratificación socioeconómica, nivel de educación, estado civil, etc.), relacionada con hábitos (exposición al humo de tabaco y nivel de actividad física en el tiempo libre) y específica para la caracterización de los atributos del sueño, incluyendo: 1) calidad, clasificada en cinco niveles como "pésima", "mala", "regular", "buena" o "excelente"; 2) realización usual de siesta; 3) duración usual del sueño principal o nocturno, calculada como la diferencia en horas entre el momento de acostarse a dormir y el momento del despertar; y 4) ronquido, clasificando su frecuencia en cinco niveles: "nunca", "casi nunca", "algunas veces", "casi siempre" o "siempre".

Los participantes también fueron evaluados por un médico del estudio que determinó antecedentes y realizó un examen físico, que incluyó la medición de peso, talla, perímetro de cintura y presión arterial, por duplicado, empleando esfigmomanómetro aneroide, con el participante en posición decúbito supino después de al menos 5 minutos de reposo. Se definió hipertensión arterial en aquellos participantes con un diagnóstico previo y que recibían tratamiento farmacológico antihipertensivo al momento de la entrevista o que tenían cifras de presión arterial $\geq 140 / 90 \mathrm{~mm} \mathrm{Hg}$ en el examen físico realizado por el estudio. Todos los participantes evaluados en la línea de base $(n=1.644)$ fueron invitados nuevamente a una visita de seguimiento con tasas de recontacto y reevaluación del 76,5\% y $64,0 \%$, respectivamente. El comité de ética de investigación de la Universidad Autónoma de Bucaramanga aprobó el protocolo de estudio.

\section{Análisis estadístico}

Se describieron las características sociodemográficas, antropométricas, de presión arterial y distribución de los atributos del sueño mediante la estimación de medias (desviación estándar [DE]) y conteos (porcentajes) para las variables continuas y discretas, respectivamente. Se estimó la incidencia acumulada (riesgo) de hipertensión arterial e intervalo de confianza del 95\% (IC95\%) empleando el método exacto. La asociación entre atributos del sueño e incidencia de hipertensión arterial se evaluó mediante la estimación de razón de disparidad (OR) empleando modelos de regresión logística crudos y ajustados por edad e índice de masa corporal (IMC). La bondad de ajuste de dichos modelos se evaluó empleando la prueba de Hosmer-Lemeshow. Para evaluar la asociación entre atributos del sueño y el cambio de presión arterial se empleó regresión lineal múltiple, incluyendo un término para la duración del seguimiento y ajustando por edad e IMC. En estos modelos se empleó estimación robusta de la varianza para corregir la correlación intra-individuo entre medidas repetidas de presión arterial.

\section{Resultados}

Se evaluaron 1.036 participantes que asistieron tanto a la visita de línea de base de la cohorte como a una de seguimiento (mediana de duración: 12,4 años; rango intercuartílico [RIC]: 11,9 - 13,0 años) y que tenían información completa del desenlace del estudio, es decir, mediciones de presión arterial y determinación de la prescripción de tratamiento farmacológico antihipertensivo. En la línea de base del estudio la media de la edad de los asistentes al seguimiento fue de 36,8 años ( $D E=8,0$ años), 63,9\% eran hombres y 75,0\% residían en barrios con estratificación socioeconómica (ESE) 1-3. La prevalencia de hipertensión arterial en dicha evaluación inicial fue 7,2\% (IC95\%: 5,7 8,8 ) con lo cual, para efectos del análisis prospectivo, 961 participantes se encontraban a riesgo de desarrollar dicha condición.

\section{Caracterización de los atributos del sueño}

En la línea de base, $266(26,1 \%)$ participantes calificaron su sueño como regular, malo o pésimo y $523(51,3 \%)$ reportaron roncar, entre los cuales, aproximadamente uno de cada 6-7 lo hacían todas las noches. La duración promedio del sueño principal durante una semana usual fue de 7,4 horas ( $D E=1,2$ horas) distribuido de la siguiente manera: $7,4 \%$ participantes reportaron dormir $<6$ horas/días, $53,7 \%$ entre 6-8 horas/días y $38,9 \% \geq 8$ horas/día. La calidad del sueño fue similar entre roncadores y no roncadores: $24,4 \%$ y $28,1 \%$ calificaron su sueño como regular/malo/pésimo, respectivamente $(p 0,177)$. Por otra parte, una mayor proporción de participantes que reportaron una duración del sueño $<6$ horas/día calificaron este como regular/malo/pésimo en comparación con quienes dormían $\geq 6$ horas/días $(36,1 \%$ vs. $25,2 \%$, respectivamente; $p 0,042$ ).

La incidencia de ronquido fue de 49,8\% (IC95\%: 45,1 $54,5)$, mientras que aproximadamente 1 de cada 10 participantes que reportaron roncar en la línea de base no lo hicieron durante la visita de seguimiento $(6,3 \%$ entre quienes reportaron roncar siempre comparado con $14,3 \%$ entre quienes reportaba hacerlo algunas veces o casi nunca). En la línea de base se observó una asociación transversal entre adiposidad y ronquido, de tal manera que quienes reportaron roncar tuvieron en promedio $2,4 \mathrm{~kg} / \mathrm{m}^{2}$ más de IMC comparados con los no roncadores $\left(27,0\right.$ vs. $24,6 \mathrm{~kg} / \mathrm{m}^{2}$, $p>0,001)$. Esta relación también se observó longitudinalmente dado que entre los no roncadores en la línea de base un mayor IMC se asoció con mayor incidencia de ronquido, independientemente de la edad y el sexo $(O R=1,08$ por $\mathrm{kg} / \mathrm{m}^{2}$, IC95\%: 1,02 - 1,14). Por otro lado, no se observó un cambio en la media de la duración del sueño principal entre la evaluación de seguimiento y la de línea de base ( 0 0,897).

\section{Relación entre atributos del sueño e hipertensión arterial}

La incidencia acumulada de hipertensión arterial fue de 15,1\% (IC95\%: 12,8 - 17,4). En los modelos crudos, mayor edad, nivel educativo equivalente a bachillerato o inferior y estar casado o en unión libre, fueron características sociodemográficas que aumentaron el riesgo de hipertensión arterial (tabla 1). El sedentarismo (OR=1,61, IC95\%: 1,09 - 1,19), los mayores niveles de adiposidad global $\left(\mathrm{OR}=1,14\right.$ por $\left.\mathrm{kg} / \mathrm{m}^{2}\right)$ y abdominal $(\mathrm{OR}=1,05$ por $\mathrm{cm})$, así como los de la presión arterial en la línea de base $(\mathrm{OR}=1,09$ por $\mathrm{mm} \mathrm{Hg}$ de sistólica y $\mathrm{OR}=1,14$ por $\mathrm{mm} \mathrm{Hg}$ de diastólica) se asociaron de manera significativa con la progresión entre normotensión e hipertensión. Entre los atributos del sueño, solamente el reporte de ronquido se asoció a la 
Tabla 1 Características de los participantes del estudio en la línea de base, de acuerdo con la incidencia de hipertensión arterial al seguimiento

\begin{tabular}{|c|c|c|c|}
\hline Característica & $\begin{array}{l}\text { No incidente } \\
(\mathrm{n}=816)\end{array}$ & $\begin{array}{l}\text { Incidente } \\
(n=145)\end{array}$ & $\begin{array}{l}\text { OR } \\
\text { (IC95\%) }\end{array}$ \\
\hline Edad & $35,5(7,9)^{*}$ & $41,2(6,5)$ & $1,11(1,08-1,14)$ \\
\hline Masculino & $520(63,7)$ & $91(62,8)$ & $0,96(0,67-1,38)$ \\
\hline ESE 1-3 & $599(74,9)$ & $108(77,1)$ & $1,13(0,74-1,73)$ \\
\hline No. Personas/habitación & $1,6(0,9)$ & $1,6(1,3)$ & $1,00(0,83-1,22)$ \\
\hline Bachillerato o inferior & $503(62,6)$ & $102(71,3)$ & $1,48(1,01-2,19)$ \\
\hline Casado/unión libre & $513(63,7)$ & $111(77,6)$ & $1,97(1,30-3,00)$ \\
\hline Fumador actual & $146(18,2)$ & $26(18,2)$ & $1,00(0,63-1,58)$ \\
\hline Sedentarismo(tiempo libre) & $551(68,3)$ & $111(77,6)$ & $1,61(1,06-2,45)$ \\
\hline IMC (kg/m²) & $25,4(3,9)$ & $27,6(3,7)$ & $1,14(1,09-1,19)$ \\
\hline Perímetro de cintura $(\mathrm{cm})$ & $86,7(11,5)$ & $92,9(12,0)$ & $1,05(1,03-1,06)$ \\
\hline \multicolumn{4}{|l|}{ Presión arterial (mm Hg) } \\
\hline Sistólica & $114,3(9,3)$ & $121,2(8,7)$ & $1,09(1,07-1,12)$ \\
\hline Diastólica & $75,3(7,0)$ & $80,0(5,2)$ & $1,14(1,11-1,19)$ \\
\hline Media & $88,3(7,2)$ & $93,8(5,8)$ & $1,15(1,11-1,19)$ \\
\hline Pulso & $39,0(6,6)$ & $41,2(6,8)$ & $1,05(1,02-1,08)$ \\
\hline Calidad del sueño (regular/mala/pésima) & $212(26,5)$ & $35(24,5)$ & $0,90(0,60-1,36)$ \\
\hline Siesta & $368(45,9)$ & $62(43,1)$ & $0,89(0,62-1,28)$ \\
\hline Ronca & $384(47,8)$ & $86(60,6)$ & $1,68(1,17-2,41)$ \\
\hline \multicolumn{4}{|l|}{ Categorías } \\
\hline Nunca & $419(52,2)$ & $56(39,4)$ & 1,00 \\
\hline Casi nunca/algunas veces & $329(41,0)$ & $72(50,7)$ & $1,64(1,12-2,39)$ \\
\hline Siempre & $55(6,9)$ & $14(9,9)$ & $1,90(1,00-3,65)$ \\
\hline Duración del sueño (horas/día) & $7,4(1,2)$ & $7,3(1,3)$ & $0,96(0,83-1,11)$ \\
\hline \multicolumn{4}{|l|}{ Categorías } \\
\hline$<6$ horas/día & $56(7,1)$ & $12(8,5)$ & 1,00 \\
\hline 6-8 horas/día & $422(53,5)$ & $77(54,6)$ & $0,85(0,44-1,66)$ \\
\hline$>8$ horas/día & $311(39,4)$ & $52(36,9)$ & $0,78(0,39-1,55)$ \\
\hline
\end{tabular}

* Los números en cada celda corresponden a medias (desviación estándar) o conteos (porcentajes) que podrían no sumar al total debido a datos perdidos.

incidencia de hipertensión arterial, mostrando un gradiente con la frecuencia percibida respecto a quienes nunca roncaban (OR=1,64, IC95\%: 1,12 - 2,39 para casi nunca/algunas veces y $O R=1,94$, IC 95\%: 1,00 - 3,65 para siempre), sin embargo, posteriormente al ajuste por edad y adiposidad dicha asociación se hizo no estadísticamente significativa (tabla 2).

\section{Relación entre atributos del sueño y cambio en los niveles de presión arterial}

Las medias de presión arterial sistólica, diastólica, media y de pulso en la línea de base fueron 117,2; 77,1; 90,4 y $40,1 \mathrm{~mm} \mathrm{Hg}$, respectivamente. Durante el seguimiento se observaron incrementos estadísticamente significativos de

Tabla 2 Modelos multivariados para la predicción de hipertensión arterial con base en los atributos del sueño determinados en línea de base

\begin{tabular}{llc}
\hline Atributo & Modelo 1*OR (IC95\%) & Modelo 2 OR (IC95\%) \\
\hline Calidad del sueño (regular/mala/pésima) & $1,15(0,74-1,80)$ & $1,14(0,73-1,80)$ \\
Siesta & $0,91(0,62-1,33)$ & $0,86(0,58-1,26)$ \\
Ronca & $0,89(0,59-1,34)$ & $0,87(0,57-1,31)$ \\
Duración del sueño (horas/día) & 1,00 & 1,00 \\
$\quad<6$ horas/día & $0,98(0,47-2,07)$ & $0,96(0,46-2,03)$ \\
$\quad$ 6-8 horas/día & $1,02(0,48-2,21)$ & $1,04(0,48-2,25)$ \\
$\quad>8$ horas/día & & \\
\hline
\end{tabular}

" Modelo de regresión logística considerando cada atributo por separado, ajustando por edad e IMC (todos los modelos tuvieron pruebas de bondad de ajuste con valores de $p>0,05)$.

$\dagger$ Modelo de regresión logística considerando simultáneamente todos los atributos del sueño, ajustando adicionalmente por edad e IMC (prueba de bondad de ajuste: $p=0,865$ ). 


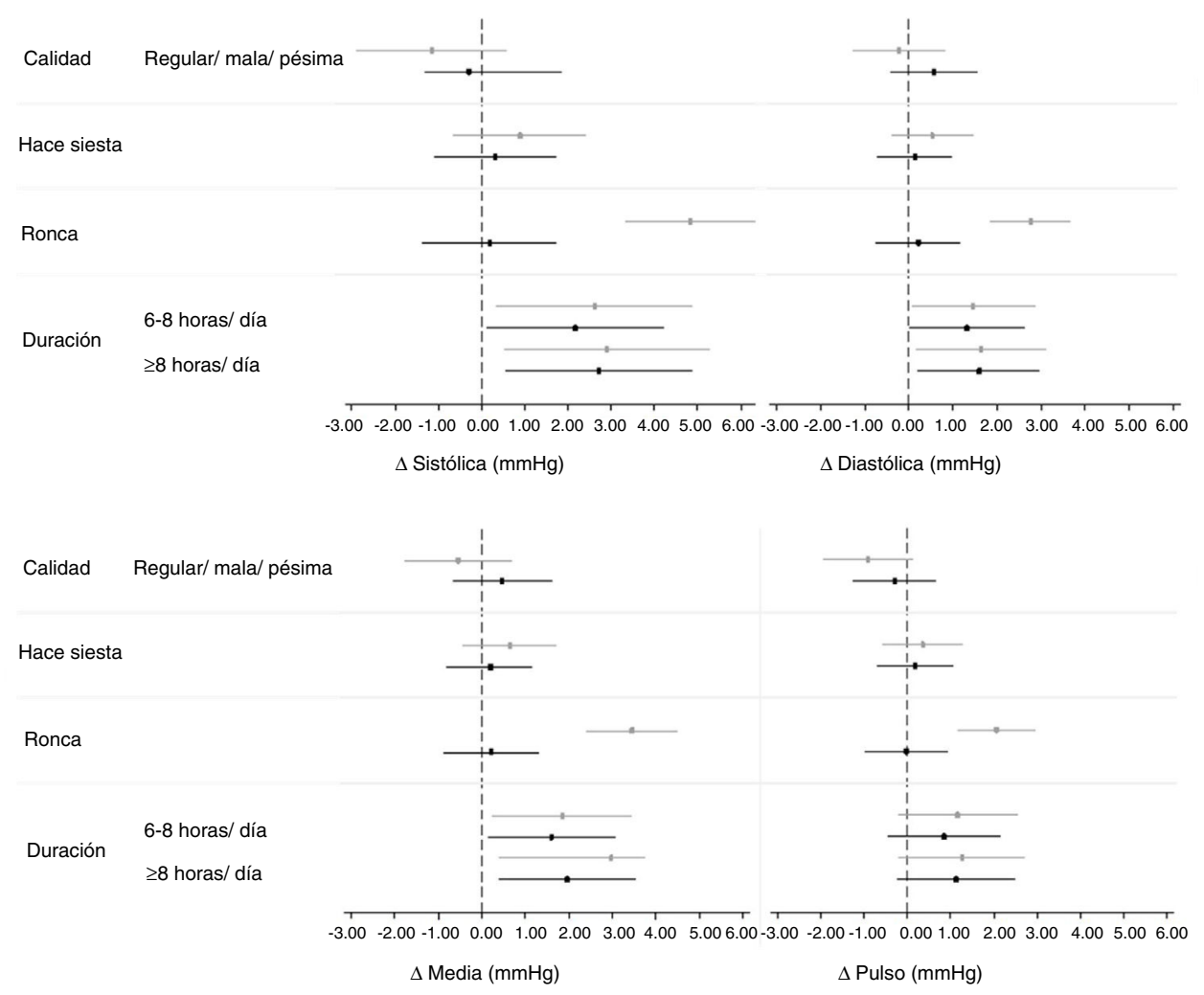

Figura 1 Cambio crudo y ajustado* de la presión arterial entre la visita de línea de base y la de seguimiento, de acuerdo con los niveles de los atributos del sueño.

* Ajustado por edad, duración del seguimiento e IMC. Calidad de sueño (referencia: buena o excelente calidad); siesta (referente: no hace siesta); ronca (referencia: nunca ronca); duración del sueño principal (referencia: <6 horas/día). En gris se presentan los estimados crudos mientras que en negro se muestran los estimados ajustados.

presión arterial sistólica $(0,97-1,91 \mathrm{~mm} \mathrm{Hg} / \mathrm{año})$, media (0,40 - 1,04 mm Hg/año) y de pulso $(0,86-1,30 \mathrm{~mm} \mathrm{Hg/año)}$ pero no de la presión arterial diastólica. Los participantes que reportaron roncar tuvieron incrementos significativos de presión arterial diastólica $(1,17 \mathrm{~mm} \mathrm{Hg}$; IC95\%: 0,22 $2,13)$ y media $(1,29 \mathrm{~mm} \mathrm{Hg}$; IC $95 \%$ : 0,19 - 2,38), comparados con los no roncadores; no obstante, dichas diferencias se atenuaron e hicieron no estadísticamente significativas posterior al ajuste por IMC (fig. 1). Finalmente, los participantes que reportaron dormir entre 6-8 horas/día y $>8$ horas/días tuvieron mayores incrementos de la presión arterial (ajustando por edad, duración del seguimiento e IMC) comparados con aquellos que reportaron duración del sueño principal <6 horas/día: 2,17 mm Hg (IC95\%: 0,12 4,22) y 2,72 mm Hg (IC95\%: 0,55 - 4,89) para la presión arterial sistólica; $1,32 \mathrm{~mm} \mathrm{Hg}$ (IC95\%: 0,00 - 2,64) y 1,59 mm Hg (IC95\%: 0,19 - 2,98) para la diastólica; y 1,60 mm Hg (IC95\%: $0,13-3,08)$ y $1,97 \mathrm{~mm} \mathrm{Hg}$ (IC95\%: 0,40 - 3,53) para la media.

\section{Discusión}

En este estudio, el primero de carácter longitudinal en que se evalúa la relación entre sueño y presión arterial en población general colombiana, se estimó que aproximadamente la mitad de los adultos sanos reportaron roncar y que uno de cada cuatro calificó su sueño principal (nocturno) como regular, malo o pésimo, con una media global de duración igual a 7,4 horas/día. Entre los no roncadores la incidencia de este hallazgo fue cercana al $50 \%$ a 12 años de seguimiento, siendo más probable en personas mayores y con mayor nivel de adiposidad. Finalmente, se halló evidencia de asociación entre roncar y una mayor duración del sueño con mayores niveles de presión arterial al seguimiento aunque en el primer caso dicha relación fue atenuada posterior al ajuste por adiposidad.

La caracterización del sueño, y más específicamente sus trastornos, en población colombiana ha sido hecha exclusivamente en el contexto de encuestas transversales ${ }^{12,13}$. Estos estudios han permitido obtener estimaciones de la prevalencia de ronquido en población general, que oscila entre 33 a 48\%, así como de la distribución de la duración del sueño nocturno, siendo similar en los residentes de Bucaramanga y los participantes de la cohorte $(2,7 \%$ vs. $2,9 \%$ reportaron $<5$ horas/día de sueño, respectivamente). Esto último sugiere que la cohorte, ensamblada a partir de donantes de banco de sangre, es representativa en cuanto a la exposición de estudio. Estos resultados, adicionalmente, aportan la primera estimación de incidencia de ronquido en el país y permiten identificar a la adiposidad, determinada mediante el IMC, como un factor de riesgo para dicha condición.

En este estudio el ronquido se asoció con mayores niveles de presión arterial e hipertensión arterial al seguimiento, sin embargo, dichas relaciones fueron atenuadas y tornadas en 
no estadísticamente significativas luego del ajuste multivariado. Estos hallazgos no concuerdan con lo reportado por la cohorte para el estudio de la salud de las enfermeras en los Estados Unidos (NHS; Nurses' Health Study) en el que el ronquido se asoció tanto con mayores niveles de presión arterial (sistólica: 1,40 - 2,13 mm Hg; diastólica: 0,78-1,15 mm Hg) como con riesgo de hipertensión arterial ( 29 y $55 \%$ en roncadoras ocasionales y regulares, respectivamente), después de 8 años de seguimiento ${ }^{14}$. Dos estudios de cohorte más, uno conducido en hombres europeos entre los 30 - 69 años con seguimiento de 10 años y otro en población asiática no obesa entre los 40 - 69 años, con seguimiento de 2 años, también reportaron la existencia de asociación entre el ronquido y la incidencia de hipertensión arterial ${ }^{15,16}$.

Frente a esta discrepancia, es interesante considerar que en la línea de base de nuestro estudio, como en las cohortes descritas, el reporte de ronquido y su frecuencia se asociaron con mayores niveles de adiposidad pero que adicionalmente este factor se relacionó con mayor incidencia de ronquido durante el seguimiento. Lo anterior podría explicar la notable atenuación observada en la relación entre antecedente de ronquido y presión arterial (e incidencia de hipertensión arterial) una vez ajustada particularmente por adiposidad. Adicionalmente, la nuestra fue una cohorte ensamblada predominantemente con adultos jóvenes (a diferencia de las otras cohortes) en quienes el incremento esperado de la presión arterial con la edad es menos acentuado, incluso durante un seguimiento de 12 años $^{17}$, lo que hace menos probable detectar diferencias.

El efecto de la duración del sueño sobre el riesgo de desarrollar hipertensión arterial ha sido previamente descrito ${ }^{5}$. Particularmente, un reciente metaanálisis de estudios longitudinales apunta a que una menor duración del sueño principal (nocturno), usualmente definido como inferior a 5 horas, se asocia con mayor incidencia de hipertensión arterial, cuando se contrasta con duraciones alrededor de las 7 horas $^{8}$. En la interpretación de estos resultados es importante considerar que en solo 1 de los 7 estudios incluidos, una duración corta del sueño se asoció estadísticamente con el desenlace ${ }^{18}$, exclusivamente en mujeres. En el mismo metaanálisis se evaluó también la relación entre sueño de larga duración e hipertensión arterial, sin embargo, los resultados no fueron concluyentes, en parte debido a la heterogeneidad de los estudios incluidos.

En nuestra cohorte no se halló evidencia de un riesgo diferencial de hipertensión arterial de acuerdo con la duración reportada del sueño en la línea de base. Esta observación concuerda con la de otro estudio longitudinal ${ }^{19}$, que tiene entre sus particularidades la recolección de información por evaluadores entrenados en el marco de una entrevista estructurada (Stanford Five-City Physical Activity Interview). De igual manera que en el caso del hallazgo negativo para ronquido, es probable que dada la edad de los participantes de nuestra cohorte se requiera de un seguimiento más prolongado que permita detectar un desenlace clínico como el diagnóstico de hipertensión arterial. Adicionalmente, la interpretación de nuestros resultados debe hacerse a la luz de la distribución de la duración del sueño en los participantes del estudio ya que menos del $3 \%$ reportaron dormir menos de 5 de horas/día, lo cual podría atenuar una probable asociación en el límite inferior de la exposición debido a mayor incertidumbre.
Por otra parte, al analizar la relación entre duración del sueño y cambio en la presión arterial se observó un gradiente, con mayor incremento en quienes reportaron duraciones de 6-8 y más de 8 horas, respecto a quienes dormían menos de 6 horas/días. Este hallazgo no es único de nuestro estudio ya que en la cohorte CARDIA, que evaluó también adultos jóvenes durante un seguimiento de 5 años, se observó una tendencia al incremento de la presión arterial sistólica en participantes con duración del sueño menor a 7 horas/días ${ }^{20}$.

Entre las fortalezas de nuestro estudio se encuentran el tener una base poblacional, su naturaleza prospectiva y una alta adherencia al seguimiento, características que, aunadas a la distribución similar de los atributos del sueño respecto a encuestas poblacionales del área urbana, confieren a los resultados mayor validez interna, dada la relación temporal entre exposición y desenlace, y mayor validez externa en cuanto a su extrapolación a la población de referencia. La recolección de información acerca de la exposición por entrevistadores entrenados y la medición directa del desenlace mediante examen clínico minimizan errores sistemáticos como los asociados al autorreporte.

Este estudio también posee limitaciones, una de las cuales es común a la mayoría de los estudios epidemiológicos acerca del sueño y tiene que ver con la determinación de esta exposición mediante cuestionario en lugar de emplear métodos más objetivos como la actigrafía o la polisomnografía, aunque estos, como bien se sabe, pueden alterar el hábito usual del sueño. Por otra parte, dado que la cohorte se ensambló con base en una muestra de adultos jóvenes, incluso con un seguimiento de cerca de una década, es probable que no se tenga el poder estadístico necesario para detectar cambios en la incidencia de enfermedades crónicas como la hipertensión arterial.

\section{Conclusión}

Este estudio aporta información novedosa acerca de la distribución de diferentes atributos del sueño en población urbana de Colombia, así como la primera estimación de la incidencia del ronquido e identificación de la adiposidad como uno de sus factores de riesgo. Ninguno de los atributos del sueño se asoció de manera independiente con la incidencia de hipertensión arterial; sin embargo, sí se halló evidencia a favor de la existencia de un gradiente entre la duración del sueño nocturno y el cambio de la presión arterial al seguimiento.

\section{Financiación}

La información de seguimiento CHICAMOCHA fue obtenida gracias a la financiación de COLCIENCIAS, contrato 489-2012.

\section{Conflicto de intereses}

Ninguno. 


\section{Agradecimientos}

Este estudio fue posible gracias a la colaboración de los bancos de sangre de Bucaramanga y los donantes de sangre en CHICAMOCHA, así como de los voluntarios participantes en las encuestas poblacionales.

\section{Bibliografía}

1. Lim SS, Vos T, Flaxman AD, Danaei G, Shibuya K, Adair-Rohani H, et al. A comparative risk assessment of burden of disease and injury attributable to 67 risk factors and risk factor clusters in 21 regions, 1990-2010: a systematic analysis for the Global Burden of Disease Study 2010. Lancet. 2012;380:2224-60.

2. Rodriguez J, Ruiz F, Peñaloza E, Eslava J, Gómez LC, Sánchez H, et al. Encuesta Nacional de Salud 2007. Resultados Nacionales. 2009. Bogotá: Fundación Cultural Javeriana de Artes Gráficas JAVEGRAF.

3. Ministerio de Salud CNdC. II Estudio Nacional de Factores de Riesgo de Enfermedades Crónicas-ENFREC II. 1999.

4. Instituto Nacional de Salud ONdS. Quinto Informe ONS: carga de enfermedad por enfermedades crónicas no transmisibles y discapacidad en Colombia. 2015. Imprenta Nacional de Colombia, Bogotá, D.C., 2015.

5. Gangwisch JE. A review of evidence for the link between sleep duration and hypertension. Am J Hypertens. 2014;27:1235-42.

6. Tochikubo O, Ideda A, Miyajima E, Ishii M. Effects of insufficient sleep on blood pressure monitored by a new multibiomedical recorder. Hypertension. 1996;27:1318-24.

7. Lusardi P, Zoppi A, Preti P, Pesce RM, Piazza E, Fogari R. Effects of insufficient sleep on blood pressure in hypertensive patients. A 24-h study. Am J Hypertens. 1999;12:63-8.

8. Meng L, Zheng Y, Hui R. The relationship of sleep duration and insomnia to risk of hypertension incidence: a meta-analysis of prospective cohort studies. Hypertens Res. 2013;36:985-95.

9. Shepard JWJ. Hypertension, cardiac arrhythmias, myocardial infarction, and stroke in relation to obstructive sleep apnea. Clin Chest Med. 1992;13:437-58.
10. Hoffstein V. Is snoring dangerous to your health. Sleep. 1996;19:506-16.

11. Villar JC, Herrera VM, Chaves AM, Martínez LX, Villar LA, Ardila E, et al., "Cardiovascular Health Investigation and Collaboration to Assess the Markers and Outcomes of Chagas disease" (CHICAMOCHA). Concepto, métodos y características de una cohorte de donantes de sangre en Bucaramanga, Colombia. MEDUNAB. 2015;18:14-26.

12. Ruiz AJ, Sepúlveda MA, Martínez PH, Muñoz MC, Mendoza LO, Centanaro OP, et al. Prevalence of sleep complaints in Colombia at different altitudes. Sleep Sci. 2016;9:100-5.

13. Díaz R, Ruano MI, Chacón A. Estudio de trastornos de sueño en Caldas. Colombia (SUECA). Acta Med Colomb. 2009;32: 66-72.

14. Hu FB, Willett WC, Colditz GA, Ascherio A, Speizer FE, Rosner $B$, et al. Prospective study of snoring and risk of hypertension in women. Am J Epidemiol. 1999;150:806-16.

15. Lindberg E, Janson C, Gislason T, Svärdsudd K, Hetta J, Boman G. Snoring and hypertension: A 10 year follow-up. Eur Respir J. 1998;11:884-9.

16. Kim J, Yi H, Shin KR, Kim JH, Jung KH, Shin C. Snoring as an Independent Risk Factor for hypertension in the nonobese population: The Korean Health and Genome Study. Am J Hypertens. 2007;20:819-24.

17. Wills AK, Lawlor DA, Matthews FE, Sayer AA, Bakra E, BenShlomo Y, et al. Life course trajectories of systolic blood pressure using longitudinal data from eight UK cohorts. PLoS Med. 2011;8:e1000440.

18. Kim SJ, Lee SK, Kim SH, Yun CH, Kim JH, Thomas RJ, Shin C. Genetic association of short sleep duration with hypertension incidence-a 6-year follow-up in the Korean genome and epidemiology study. Circ J. 2012;76:907-13.

19. Hall MH, Muldoon MF, Jennings JR, Buysse DJ, Flory JD, Manuck SB. Self-reported sleep duration is associated with the metabolic syndrome in midlife adults. Sleep. 2008;31:635-43.

20. Knutson KL, Van Cauter E, Rathouz PJ, Yan LL, Hulley SB, Liu K, et al. Association between sleep and blood pressure in midlife: the CARDIA sleep study. Arch Intern Med. 2009;169: 1055-61. 